\title{
Prognostic Significance of Immunohistochemical Expression of Chemokine Receptor (CXCR4) and RKIP in Gastric Carcinoma and Premalignant Lesions of the Stomach
}

Mona A. Abo El-Khair, Mohebat H. Gouda, Adel Z. Elseaidy, Magda H. Bakr, Rasha M. Abdrabh

\begin{abstract}
Department of pathology, Faculty of Medicine Benha University, Egypt.
\end{abstract}

Correspondence to: Mona A. Abo El-Khair, Department of pathology, Faculty of Medicine Benha University, Egypt.

Email:

mona_ahmed26777@yahoo.com

Received: 13 December 2020

Accepted: 1 September 2021

\section{Abstract:}

Background: CXCR4 and RKIP have been implicated in initiation and progression in many cancers, but their role in gastric adenocarcinoma remains vague. Aim: The aim of this work is to assess the possible significance of both markers in gastric adenocarcinoma and premalignant lesions of the stomach. Methods: This retrospective study was carried upon 50 cases of gastric adenocarcinoma and 20 of premalignant lesions. Immunohistochemistry was performed to examine the expression of CXCR4 and RKIP in both gastric adenocarcinoma and premalignant lesions of the stomach. Results: CXCR4 was found to be highly expressed in gastric adenocarcinoma compared to premalignant lesions ( $\mathrm{P}$ value $=0.027)$. It was significantly correlated with tumor grade $(\mathrm{P}$ value $=0.027)$, depth of tumor invasion ( $\mathrm{P}$ value $=0.019)$, lymph node metastasis $(\mathrm{P}$ value $=0.025)$, distant metastasis $(\mathrm{P}$ value $=0.044)$ and TNM stage $(\mathrm{P}$ value $=0.002)$. No statistically significant correlation between CXCR4 expression and histopathological types (P value=0.8). RKIP expression has no significant correlation with progression from premalignant gastric lesions to adenocarcinoma $(\mathrm{P}$ value=0.078). RKIP was negatively associated with advanced tumor grade $(\mathrm{P}$ value=0.001), depth of invasion $(\mathrm{P}$ value $=0.018)$, distant metastasis $(\mathrm{P}$ value $=0.029)$ and TNM stage $(\mathrm{P}$ value=0.007). No statistically significant correlation between RKIP expression and histopathological types (P value $=0.17)$ and lymph node status $(\mathrm{P}$ value $=0.053)$. No significant correlation between $\mathrm{CXCR} 4$ and RKIP expression in studied cases was detected ( $\mathrm{P}$ value=0.178). Conclusions: These results suggested that CXCR4 might be involved in gastric carcinogenesis.

Keyword: Gastric adenocarcinoma, premalignant, CXCR4, RKIP. 


\section{Introduction}

Gastric carcinoma (GC) is the $5^{\text {th }}$ most common malignancy worldwide and remains the third cause of death as it was estimated that GC was responsible for about 783,000 deaths in 2018 worldwide (1).

In Egypt, Gastric cancer is in the $11^{\text {th }}$ rank constituting $2.1 \%$ of all cancers with slight male predilection. (2). The natural sequence of GC is well known and preceded by progressive histopathological changes that begin with acute gastritis then chronic gastritis that can lead to atrophic gastritis and progress to intestinal metaplasia, low grade dysplasia, high grade dysplasia and finally adenocarcinoma (3).

Chemokines are small secreted proteins, best known for their vital roles in mediating immune cell trafficking and can be further subdivided into four main subclasses depending on the location of the first two cysteine residues in their protein sequence (4).

Several studies showed that CXCR4 has important roles in cell death and survival depending on the cellular context. It was found to be over-expressed in about many tumors including lung, breast, liver, colorectal, bladder, and ovarian cancers (5).
Raf kinase inhibitor protein (RKIP) is a kinase inhibitor protein that regulates many signaling pathways within the cell and it was found that its downregulation may influence metastasis, angiogenesis and genome integrity (6).

The aim of this study is to evaluate the IHC expression of CXCR4 and RKIP in both gastric adenocarcinoma and premalignant lesions of the stomach, to clarify its diagnostic and prognostic role.

\section{Material and Methods}

This retrospective study was carried upon50 cases of gastric adenocarcinoma and 20 cases of premalignant lesions which were archival formalin-fixed, paraffin embedded blocks and processed during the years 2015-2020 from the Pathology Department of Benha faculty of medicine. Studied cases were endoscopic biopsies, partial and total gastrectomy specimens. The study was approved by the Research Ethics committee.

Histopathological study: Histopathological analysis was carried out on H\&E-stained sections. Gastric adenocarcinoma cases were assessed regarding the histopathological type (7), the grade and TNM stage. Canadian Cancer Society classified gastric carcinoma into low grade (grade I and grade II) and high grade (Grade III). Gastric adenocarcinomas were staged by TNM staging to stage I, II, 
III, IV. Concerning dysplastic lesions, dysplasia was classified as low grade and high grade (8).

Immunohistochemical study:

According to manufacture instructions, 3-4micron tissue sections were obtained from formalin-fixed, paraffin-embedded tissue blocks on coated slides. After xylene deparaffinization, the sections were rehydrated in descending grades of alcohol then in distilled water. Antigen retrieval was done by using $10 \mathrm{mmol} / \mathrm{L}$ citrate monohydrate buffer $(\mathrm{pH}$ 6.0) and heated for 15 minutes in microwave. Slides then were immunostained for anti-CXCR4 (Chongqing,YPA1754,China) and anti RKIP (Chongqing,YPA1916,China) with concentration 1:200 for both markers and slides were incubated at $4 \mathrm{C}$ overnight; were applied to each section. Immunodetection was executed using a standard labeled streptavidin-biotin system

(DakoCytomation, Denmark, A/S).

-Freshly prepared chromogen diaminobenzine (DAB) was used; it was incubated with slides for 3-5 minutes then washed with distilled water.

-Negative control: Omitting of primary antibody during staining was used as negative control -Positive control: Tissues from human breast cancer was used as positive control for CXCR4 and human prostatic cancer for RKIP.

- Immunohistochemical assessment:

CXCR4 staining was cytoplasmic and according to Okuyama Kishima et al (9) criteria for scoring CXCR4 expression, the intensity of cytoplasmic staining graded into: 0 (no staining), 1 (weak), 2 (moderate) and 3 (intense). The percentage of positive tumor cells was scored as 0 (negative), 1 (<10\%), 2 $(10 \%-50 \%), 3(>50 \%)$. Staining index was calculated by multiplying the intensity by the percentage of positive cells. Staining scores 0 and 1 are considered to be negative while scores from 2 to 9 are considered to be positive.

Expression of RKIP is cytoplasmic. The intensity of cytoplasmic staining varied from weak to strong: 0 (no staining), 1 (weak), 2 (moderate) and 3 (intense). The percentage of positive tumor cells was scored as 0 (negative), 1 (<10\%), $2(10 \%-50 \%), 3$ $(>50 \%)$. Staining score was calculated by multiplying the intensity score by the extent of positive cells. Scores were further grouped into two categories: negative $($ scores $<4)$ and positive (scores $\geq 4$ ) (10).

\section{Statistical analysis:}

Results were analyzed using SPSS (version 16). The Pearson correlation coefficient was 
used for statistical analysis. $\mathrm{P}$ value $<0.05$ was considered statistically significant (*) and highly statistically significant(**) when it was $<0.01$. ROC curve was used to predict sensitivity, specificity and accuracy to detect validity of CXCR4 to predict non neoplastic group from adenocarcinoma cases and the validity of RKIP in prediction of distant metastasis.

\section{Results:}

Premalignant lesions included 8 cases of chronic gastritis, 6 cases of chronic gastritis with intestinal metaplasia and 6 cases of chronic gastritis with dysplasia (low and high grades).

Adenocarcinoma studied cases were classified into 26 cases of tubular adenocarcinoma (52\%), 13 cases of mucinous adenocarcinoma (26\%) and $11(22 \%)$ cases were of signet ring type. They were graded into: $27(54 \%)$ cases of low grade (I\&II) and 23 (46\%) cases of high grade gastric adenocarcinoma (III). According to tumor depth of invasion, cases were classified into: 6 (12\%) cases were T1, $13(26 \%)$ cases were T2, $18(36 \%)$ cases were T3 and $13(26 \%)$ cases were T4. Out of the 50 adenocarcinoma cases, $14(28 \%)$ cases were N0, 14 (28\%) cases were N 1, 13 (26\%) were N2 and 9 (18\%) cases were N3. Thirty- one $(62 \%)$ cases were M0 and the other 19 (38\%) cases were M1. Also, they were staged according to TNM staging into; 7 (14\%) cases were stage I,13(26\%) cases were stage II, 11(22\%) were stage III while 19(38\%) were stage IV. Histopathological results are shown in fig.1.

\section{Immunohistochemical Results:}

Out of 20 premalignant lesions cases, 7 cases (35\%) were positive for CXCR4 staining and 13 cases (65\%) were negative for CXCR4 staining, so CXCR4 expression was significantly increased gradually with gastric disease progression from different premalignant gastric lesions to adenocarcinoma (p value $<\mathbf{0 . 0 1}$ ). There was no significant correlation $(P$ value $=0.8)$ between CXCR4 expression \& different histopathological types of gastric adenocarcinoma. There was a statistically significant positive correlation between CXCR4 expression score and advanced tumor grade $(\mathbf{P}$ value $=\mathbf{0 . 0 2 7})$, depth of invasion $(\mathbf{P}$ value $=\mathbf{0 . 0 3 5})$, lymph node status $(\mathbf{P}$ value $=\mathbf{0 . 0 2 5})$, distant metastasis $(\mathbf{P}$ value $=\mathbf{0 . 0 4 4}$ ) and $\mathrm{TNM}$ stage of gastric carcinoma $(\mathbf{P}$ value $=\mathbf{0 . 0 0 2})$. $\mathrm{CXCR} 4$ results are viewed in fig.2. Out of 20 Premalignant cases, 13 cases $(65 \%)$ were positive for RKIP while 7 cases $(35 \%)$ were negative for RKIP 
staining, so RKIP expression has no significant correlation with progression from different premalignant gastric lesions to adenocarcinoma ( $\mathbf{P}$ value $\mathbf{0 . 0 7 8}$ ). Also, there was no significant correlation (P value =0.09) between RKIP expression \& different histopathological types of gastric adenocarcinoma and lymph node metastasis ( $P$ value $=\mathbf{0 . 0 5 3})$. There was a statistically significant negative correlation between RKIP expression and advanced tumor grade $(\mathbf{P}$ value $=\mathbf{0 . 0 2 7})$, depth of tumor invasion $(\mathbf{P}$ value $=\mathbf{0 . 0 4 3}$ ), the distant metastasis of gastric carcinoma $(\mathbf{P}$ value $=\mathbf{0 . 0 1})$ and $\mathrm{TNM}$ stage of gastric carcinoma $(\mathbf{P}$ value $\mathbf{= 0 . 0 0 2})$. RKIP results are viewed in fig. 3

No significant statistical correlation between CXCR4 and RKIP expression in studied gastric non neoplastic and adenocarcinoma cases $(\mathrm{P}$ value $=0.178)$.

The validity of CXCR4 to predict non neoplastic group from adenocarcinoma cases and validity of RKIP in prediction of distant metastasis are shown in Fig.4.

The results of both markers were correlated with different clinicopathological data of the examined cases and viewed in table 1.

Table (1): Shows the relationship between CXCR4 and RKIP Immunohistochemical results and different clinico-pathological data of studied cases.

\begin{tabular}{|c|c|c|c|c|c|c|c|}
\hline \multicolumn{2}{|c|}{ Clinico-pathological parameters } & \multicolumn{2}{|c|}{ CXCR4 expression } & \multirow{2}{*}{$\begin{array}{l}\mathbf{P} \\
\text { value } \\
\end{array}$} & \multicolumn{2}{|c|}{ RKIP expression } & \multirow[t]{2}{*}{ P value } \\
\hline & & Negative & Positive & & Negative & Positive & \\
\hline \multirow[t]{2}{*}{ Studied cases } & Premalignant lesions & $13(65 \%)$ & $7(35 \%)$ & \multirow[t]{2}{*}{$0.027 *$} & $7(35 \%)$ & $13(65 \%)$ & \multirow[t]{2}{*}{0.078} \\
\hline & Gastric adeno carcinoma & $18(36 \%)$ & $32(64 \%)$ & & $27(54 \%)$ & $23(46 \%)$ & \\
\hline \multirow{3}{*}{$\begin{array}{l}\text { Histopathological } \\
\text { subtypes of gastric } \\
\text { adenocarcinoma }\end{array}$} & Tubular adenocarcinoma & $9(34.6 \%)$ & $17(65.4 \%)$ & \multirow[t]{3}{*}{0.8} & $13(50 \%)$ & $13(50 \%)$ & \multirow[t]{3}{*}{0.17} \\
\hline & Mucinous adenocarcinoma & $5(38.5 \%)$ & $8(61.5 \%)$ & & $6(46 \%)$ & $7(54 \%)$ & \\
\hline & Signet ring adenocarcinoma & $4(36.4 \%)$ & $7(63.6 \%)$ & & $8(72.7 \%)$ & $3(27.4 \%)$ & \\
\hline \multirow{2}{*}{$\begin{array}{l}\text { Grade of gastric } \\
\text { adenocarcinoma }\end{array}$} & Low grade(I\&II) & $13(48 \%)$ & $14(52 \%)$ & \multirow[t]{2}{*}{$0.027 *$} & $9(33.3 \%)$ & $18(66.7 \%)$ & \multirow{2}{*}{$\begin{array}{l}0.001^{` *} \\
*\end{array}$} \\
\hline & High grade (III) & $5(21.7 \%)$ & $18(78.3 \%)$ & & $18(78.3 \%)$ & $5(21.7 \%)$ & \\
\hline \multirow[t]{4}{*}{ Depth of invasion } & T1 & $3(50 \%)$ & $3(50 \%)$ & \multirow[t]{4}{*}{$0.019^{*}$} & $3(50 \%)$ & $3(50 \%)$ & \multirow[t]{4}{*}{$0.018^{*}$} \\
\hline & T2 & $7(53.8 \%)$ & $6(46.2 \%)$ & & $3(23 \%)$ & $10(77 \%)$ & \\
\hline & T3 & $7(38.9 \%)$ & $11(61.1 \%)$ & & $11(61 \%)$ & $7(39 \%)$ & \\
\hline & T4 & $1(7.7 \%)$ & $12(92.3 \%)$ & & $10(77 \%)$ & $3(23 \%)$ & \\
\hline \multirow{4}{*}{ Lymph node status } & No & $6(42.8 \%)$ & $8(57.2 \%)$ & \multirow[t]{4}{*}{$0.025^{*}$} & $5(35.7 \%)$ & $9(64.3 \%)$ & \multirow[t]{4}{*}{0.053} \\
\hline & N1 & $9(64.3 \%)$ & $5(35.7 \%)$ & & $9(64.3 \%)$ & $5(35.7 \%)$ & \\
\hline & $\mathbf{N 2}$ & $2(15.4 \%)$ & $11(84.6 \%)$ & & $7(53.8 \%)$ & $6(46.2 \%)$ & \\
\hline & N3 & $1(11.1 \%)$ & $8(88.9 \%)$ & & $6(66.7 \%)$ & $3(33.3 \%)$ & \\
\hline \multirow[t]{2}{*}{ Distant Metastasis } & M0 & $14(45.2 \%)$ & $17(54.8 \%)$ & \multirow[t]{2}{*}{$0.044 *$} & $13(42 \%)$ & $18(58 \%)$ & \multirow[t]{2}{*}{$0.029 *$} \\
\hline & M1 & $4(21 \%)$ & $15(79 \%)$ & & $14(73.7 \%)$ & $5(27.3 \%)$ & \\
\hline \multirow{4}{*}{$\begin{array}{l}\text { TNM stage of gastric } \\
\text { adenocarcinoma }\end{array}$} & Stage I & $6(85.7 \%)$ & $1(21 \%)$ & \multirow{4}{*}{$\begin{array}{l}0.002 * \\
*\end{array}$} & $2(28.5 \%)$ & $5(71.5 \%)$ & \multirow[t]{4}{*}{$0.007 * *$} \\
\hline & Stage II & $6(46 \%)$ & $7(54 \%)$ & & $5(38.5 \%)$ & $8(61.5 \%)$ & \\
\hline & Stage III & $2(18 \%)$ & $9(82 \%)$ & & $6(54.5 \%)$ & $5(45.5 \%)$ & \\
\hline & Stage IV & $4(21 \%)$ & $15(79 \%)$ & & $14(73.7 \%)$ & $5(26.3 \%)$ & \\
\hline
\end{tabular}




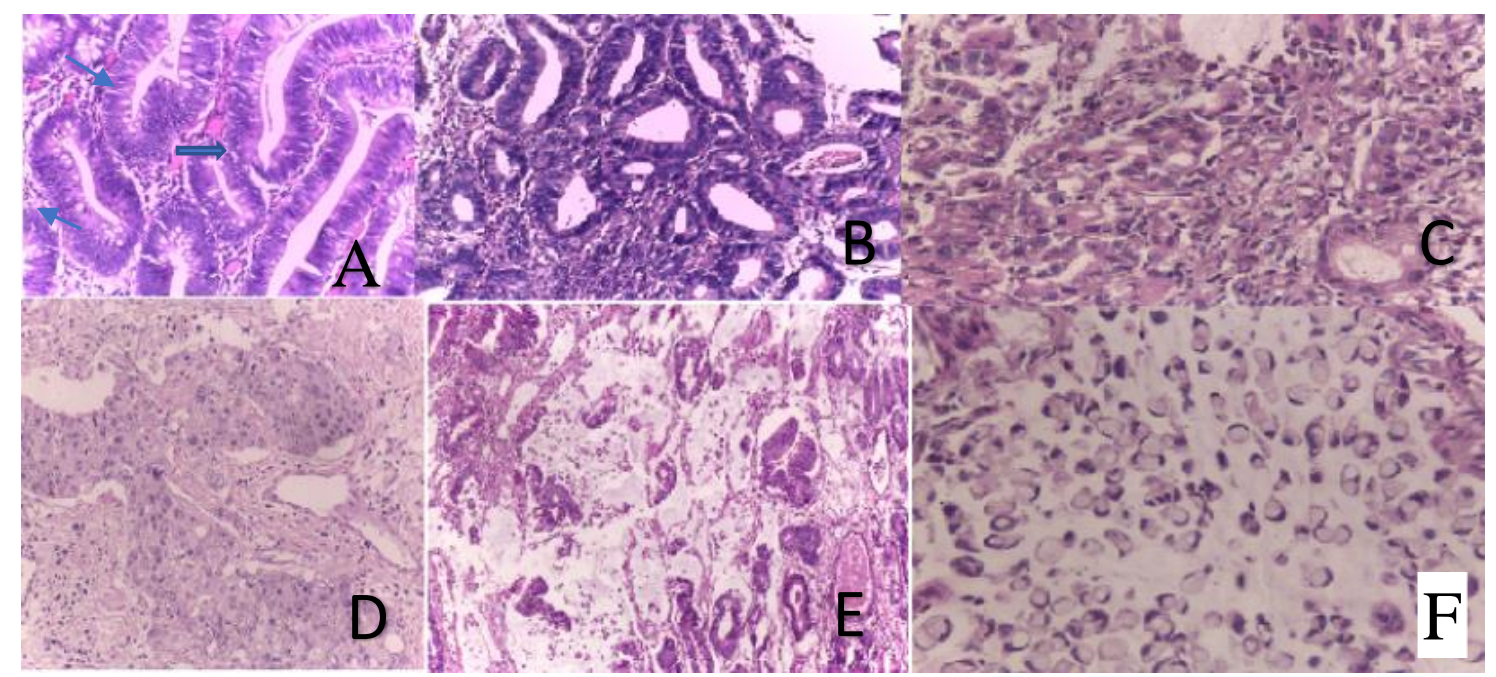

Fig. (1): A) Chronic gastritis with incomplete intestinal metaplasia with goblet cells, with focal dysplastic changes (H\&EX400). B) Well differentiated tubular gastric adenocarcinoma showing well formed glands in which nuclei are pleomorphic in size and shape and hyperchromatic (H\&EX200). C) Moderately differentiated tubular adenocarcinoma (H\&EX200). D) Poorly differentiated tubular carcinoma, grade III showing solid sheets of malignant cells with absence of glandular pattern, high grade nuclear anaplasia and increased mitotic figures (ABC X400). E) Moderately differentiated mucinous gastric adenocarcinoma showing irregular clusters of malignant cells floating in pools of mucin, moderately differentiated (H\&EX200). F) Signet ring adenocarcinoma, grade III intracellular mucin accumulation displacing the nucleus giving the signet ring appearance (H\&EX400).

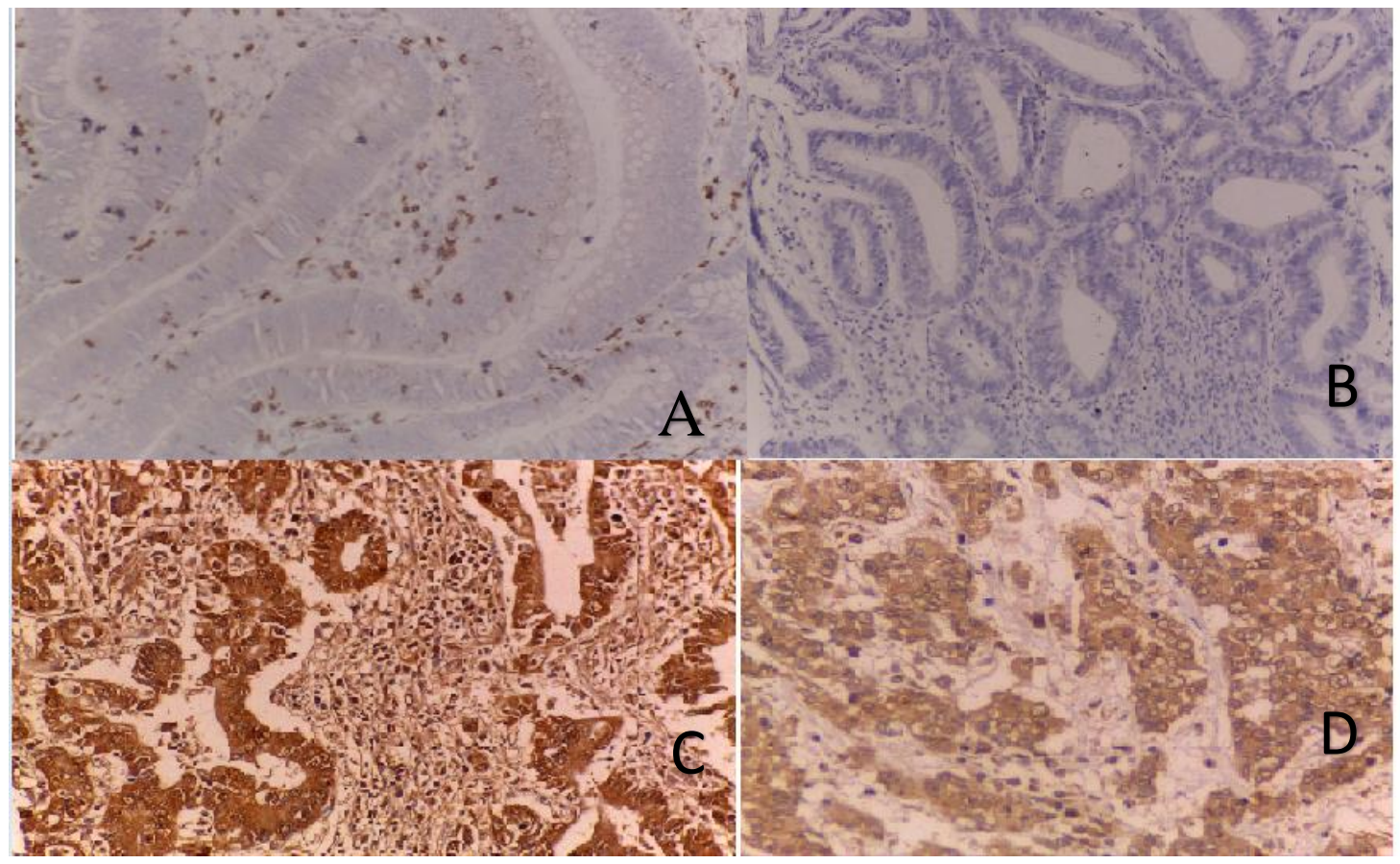

Fig. (2): A) Chronic gastritis with intestinal and focal low grade dysplasia showing negative cytoplasmic staining, for CXCR4 (ABCX400). B) Well differentiated tubular gastric adenocarcinoma showing negative cytoplasmic staining for CXCR4 (ABCX400). C) Moderately differentiated tubular carcinoma, showing positive cytoplasmic staining for CXCR4 (ABCX200). D) Poorly differentiated tubular carcinoma, grade III showing positive cytoplasmic staining for CXCR4 (ABCX400). 


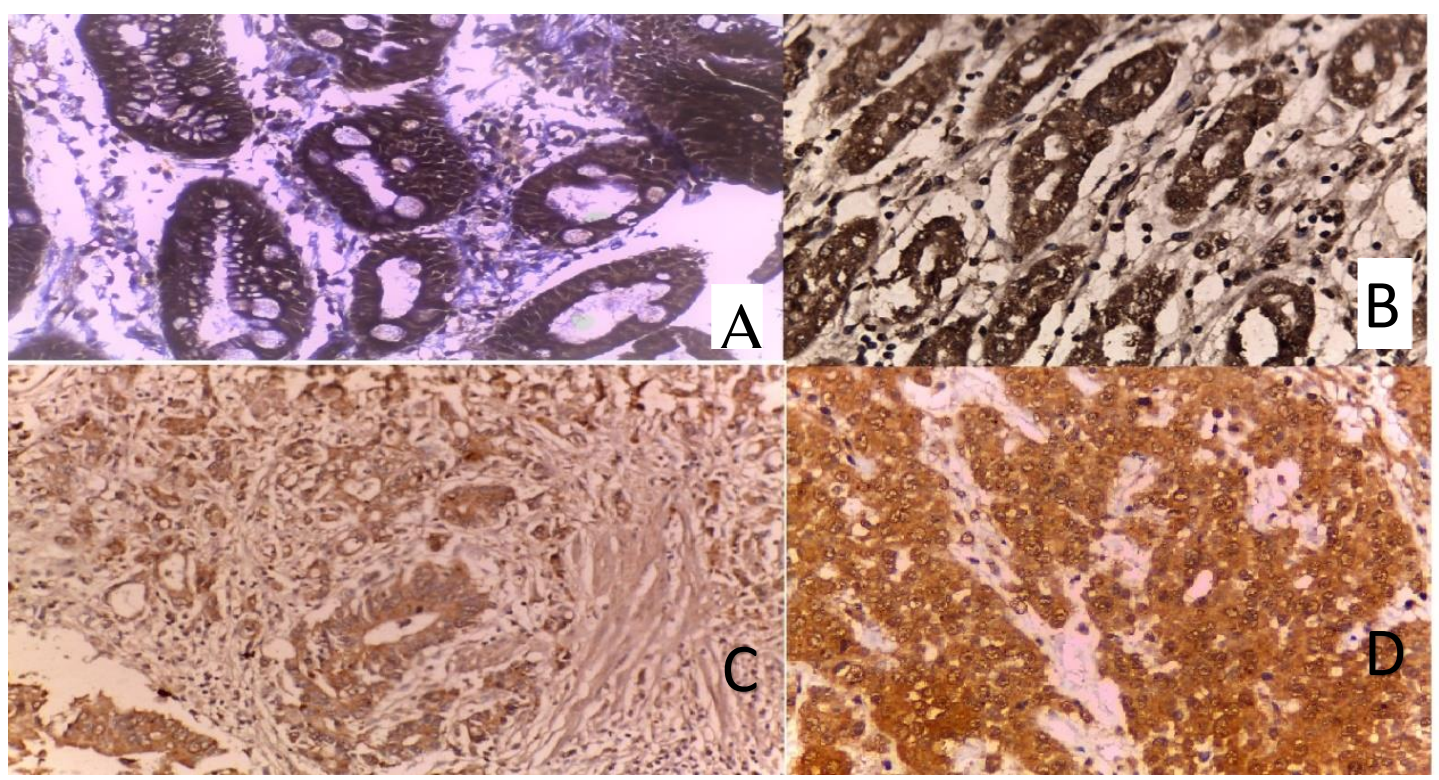

Fig. (3): A) Chronic gastritis with incomplete intestinal metaplasia showing positive cytoplasmic staining, for RKIP (ABCX400). B) well differentiated gastric adenocarcinoma showing positive cytoplasmic staining, score (9+) for RKIP (ABC X200).C) Moderately differentiated gastric adenocarcinoma showing positive cytoplasmic staining, score (9+) for RKIP (ABC X200). D) Poorly differentiated gastric adenocarcinoma showing negative cytoplasmic for RKIP (ABC X200).
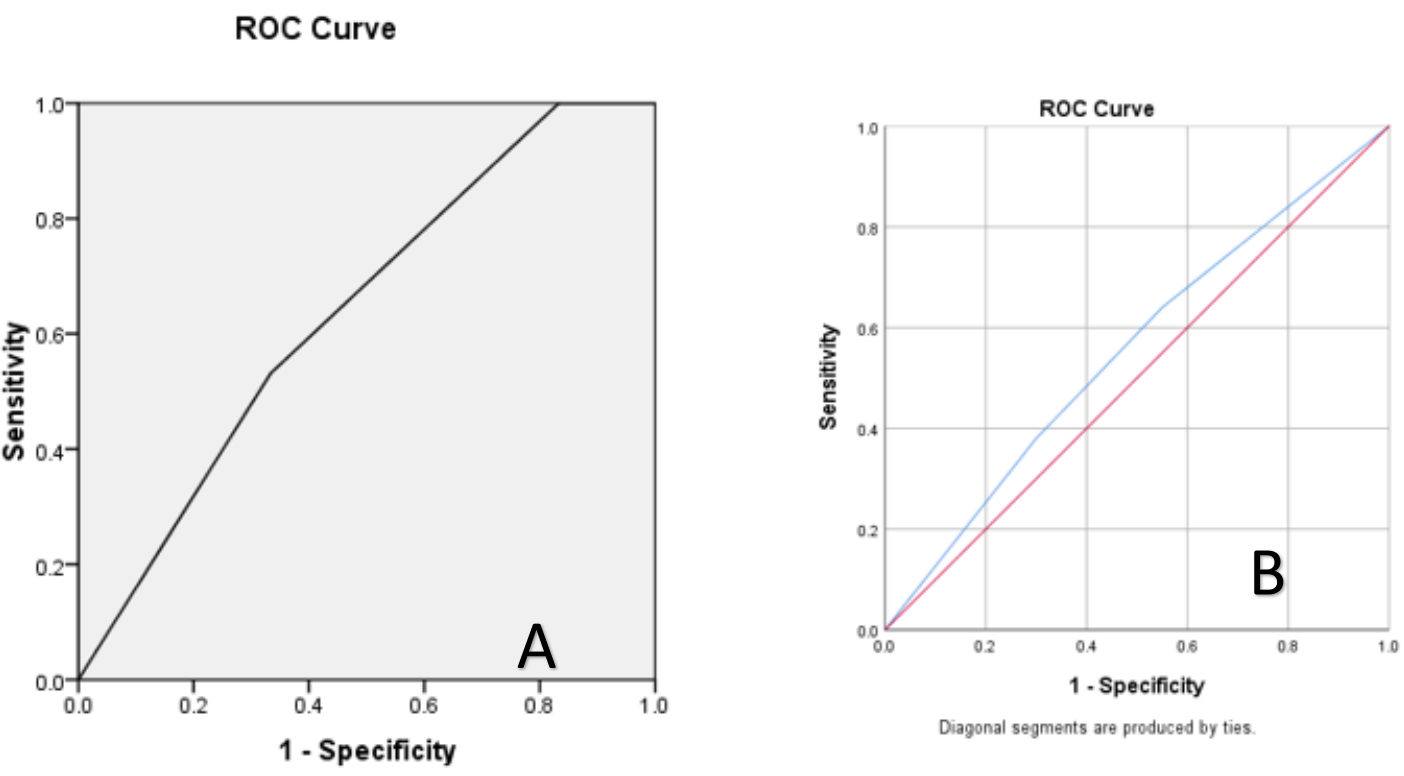

Diagonal segments are produced by ties.

Diagonal segments are produced by ties.

Fig. (4): A) Validity of CXCR4 to predict premalignant group from adenocarcinoma group: AUC of CXCR4 was 0.688 (Fair) for prediction of gastric adenocarcinoma. Sensitivity of CXCR4 (True positive cases) was 64 and Specificity of CXCR4 (True negative cases) was 65. Positive predictive value was 82.1 and negative predictive value was 41.9.

B): Validity of RKIP in prediction of distant metastasis. AUC of RKIP was 0.662 (Fair) for detection of distant metastasis. Sensitivity of RKIP (True positive cases) was 64 and Specificity of RKIP (True negative cases) was 45 . Positive predictive value was 74.4 and negative predictive value was 33.3 . 


\section{Discussion}

Gastric carcinoma (GC) is the $5^{\text {th }}$ most common cancer worldwide and remains the $3^{\text {rd }}$ cause of death as it was estimated to be responsible for about 783,000 deaths worldwide in 2018 (1).

CXCR4 was found to be important for viral entry and developing therapeutic agents for HIV-1 infection and found to be overexpressed in many tumors including lung, breast, liver, colorectal, bladder, and ovarian cancer (5).

In the present work, CXCR4 expression significantly increased gradually with progression from different premalignant lesions to gastric adenocarcinoma $(\mathbf{P}$ value $=$ 0.027). These results indicate that up regulation of CXCR4 may have an important role in the pathogenesis of gastric carcinoma.

These results were parallel to the results provided by studies made by others who used qRT-PCR technique and found that CXCR4 protein expression was highly expressed in gastric cancer than premalignant lesions (2.4 times higher). (11)

In more than a research $(12,13 \& 14)$ it was demonstrated that CXCR4 in the oral squamous cell carcinoma, endometrial and cervical cancers was significantly higher than in their premalignant lesions respectively.

These results can be explained by data which found that high levels of hypoxia in gastric carcinoma cells compared to premalignant lesions which is the responsible activation of hypoxia inducible factor 1 (HIF1) may also promote expression of a number of target genes including CXCR4 (15). CXCR4 in turn mediates rapid phosphorylation of extracellular signalregulated kinase (ERK) which suppresses apoptotic signals of caspase-9, caspase-3, and Bcl-2 and subsequently contributes to the initiation, proliferation and survival of cancer cells (12).

There was no significant correlation ( $\mathbf{P}$ value =0.8) between CXCR4 expression \& different histopathologic types of gastric adenocarcinoma. These results are similar to other results (16). However, in a different (17) it was found that CXCR4 mRNA expression was significantly higher in signet ring adenocarcinoma than other histopathological types. This disparity may be caused by difference in number of the studied cases and different techniques used. In this current study, there was a statistically significant positive correlation between 
CXCR4 expression and advanced tumor grade $(\mathbf{P}$ value $\mathbf{= 0 . 0 2 7})$

These results were parallel to others (18) where it was demonstrated that $\mathrm{CXCR} 4$ expression was related to poor differentiation of cancer cells. Data provided before (19) demonstrated that $\mathrm{CXCR} 4$ expression was significantly correlated with histopathological differentiation of endometrial adenocarcinoma and cervical adenocarcinoma respectively. However, it was also found that well differentiated gastric adenocarcinoma showed stronger CXCR4 expression than the poorly differentiated cases (20).

Our results also can be owed to high levels of HIF-1 found in advanced tumor grades which in turn upregulates CXCR4 expression (21). There was a statistically positive significant correlation between CXCR4 expression \& depth of invasion of gastric carcinoma (P value $\mathbf{= 0 . 0 3 5}$ ). These results are parallel to the data provided by some researchers (22). CXCR4 expression levels were associated with deep myometrial invasion in endometrial carcinoma as stated previously (23). However, a study found no significant correlation between CXCR4 expression \& depth of invasion of gastric carcinoma (24).
These finding can be explained by some in vitro studies that demonstrated that CXCL12/CXCR4 induces epithelialmesenchymal transition (EMT) and promotes migration in gastric cancer cells, which is accompanied by c-MET activation (12).

This work revealed that there was a statistically significant correlation between CXCR4 expression and the lymph node status in gastric adenocarcinoma ( $P$ value =0.025). These results were similar to previously reported results (25) where it was found that CXCR4 mRNA was significantly upregulated in cases of gastric adenocarcinoma with lymph node metastasis. On the contrary, there was a suggestion that there was under expression of CXCR4 inpatients with axillary lymph node metastasis in patients with ductal carcinoma of the breast (26).

Our research revealed that there was a statistically significant correlation between CXCR4 expression and the distant metastasis in gastric carcinoma ( $P$ value $=\mathbf{0 . 0 4 4})$. These results were similar to data (27) recently proving that $\mathrm{CXCR} 4$ is a key signaling molecule for metastasis of gastric cancer and with the study done previously (28) that identified CXCR4-positive gastric cancer stem cells can penetrate gastric wall, migrate 
to peritoneum with formation of omental nodules and malignant ascites in a mouse model.

There was a highly statistically significant positive correlation between CXCR4 expression \&TNM stage of gastric adenocarcinoma $(\mathbf{P}$ value $=\mathbf{0 . 0 0 2})$. These results are parallel to the results before (29), but the results provided by others (30) showed that there was no statistically significant correlation between CXCR4 expression score \&TNM stage of gastric adenocarcinoma. This conflict may be due to difference in number of studied cases.

The study done in 2014 (31) reported that CXCR4 expression was strongly correlated with lymph node metastasis, liver metastasis and TNM stage of colorectal carcinoma.

These findings can be explained by that the binding of CXCR4 to its ligand (CXCL12) can stimulate the activation of several signaling pathways that regulate the progression and metastasis of many tumors including the mitogen-activated protein kinase (MAPK) and phosphoinositide 3kinase pathways (PI3K). Their binding of on tumor cells leads also to promotion of the epithelial-to-mesechymal transition through the Rho-ROCK pathway and alterations in some cell adhesion molecules (32).
CXCR4 was found to be fair for prediction premalignant group from adenocarcinoma cases by using ROC curve. These results were near to other results (33 and 34).

A variety of evidence suggests that RKIP expression levels are frequently downregulated in various cancer types, and correlate with an invasive or metastatic phenotype (5).

In this study, RKIP expression has no significant correlation with progression from different premalignant gastric lesions to gastric adenocarcinoma ( $\mathbf{P}$ value 0.078$)$.

These results were parallel to the results of the study done in 2018 (35). This was against others (36) who stated that RKIP was significantly reduced in gastric adenocarcinoma compared to premalignant lesions and the study performed then (37) where qRT-PCR and Western blot analysis were used and proved that RKIP mRNA and protein expression was significantly downregulated in breast cancer tissues compared with the adjacent normal tissues.

In this study, we found that there was no significant correlation $(\mathbf{P}$ value $=0.09)$ between RKIP expression \& different histopathological types of gastric 
adenocarcinoma. These results are similar to former results (38). However, in the study done on 2013 (36) found that RKIP was differently expressed between the different histopathological types being highly expressed in tubular subtype compared to mucinous and signet-ring adenocarcinomas. This conflict may be caused by the difference in geographic distribution or genetic constitution of gastric carcinoma.

In this current study, there was a statistically significant negative correlation between RKIP expression and advanced tumor grade $($ P value $=0.027)$.

These results are parallel to the results provided by other studies $(39 \& 40)$ which detected that RKIP expression was detected in 58,52 and $26.8 \%$ of cases in the well, moderately and poorly differentiated groups respectively. Furthermore, the expression of RKIP was negatively associated with the histological grade of endometrial adenocarcinoma as proved by previously performed studies (41\& 42) who used PCR beside cell culture techniques found that the expression of RKIP protein decreased with the reducing differentiation of colorectal adenocarcinoma.
However, it was found that RKIP expression was not correlated to the tumor grade (43). These findings can be explained by the role of RKIP as a physiological inhibitor of the Raf-mitogen-activated protein kinase (MEK) (44). It also mediates the cross talk between many important cellular signaling pathways including the nuclear factor $\mathrm{kB}(\mathrm{NF}-\mathrm{kB})$ pathway and the $\mathrm{G}$ protein pathway. RKIP overexpression can also result in direct activation of pro-caspase 8 which plays a golden role in the execution phase of cell apoptosis (45).

There was a statistically negative significant correlation between RKIP expression \& depth of invasion of gastric adenocarcinoma $(P$ value $=\mathbf{0 . 0 4 3})$. These results are parallel to the results stating that the expression of RKIP in gastric cancer stem cells was suppressed in gastric adenocarcinoma with deep invasion (34).

In a study, it was demonstrated that low RKIP expression was associated with deep muscle invasion of urothelial carcinoma (46), and it was also found that RKIP expression was lost with deep myometrial invasion of endometrial adenocarcinoma (41). This is probably due to ERK or MEK activation following downregulation of a group of protein kinases including Raf-1(47). 
This work showed that there was no statistically significant correlation between RKIP expression and the lymph node status in gastric carcinoma $(\mathbf{P}$ value $=\mathbf{0 . 0 5 3})$.

These results were parallel to results of some researches (35), but conflicted by results of others (48) where it was found that RKIP expression was downregulated in gastric carcinoma with lymph node metastasis.

This work stated that there was a statistically significant negative correlation between RKIP expression and the distant metastasis of gastric carcinoma $(\mathbf{P}$ value $=\mathbf{0 . 0 1})$ and $\mathrm{TNM}$ stage of gastric carcinoma $(P$ value $=\mathbf{0 . 0 0 2})$. These results were similar to results reported before (49).

These results could be explained by that RKIP also negatively associated with invasion of the different cancer cells by controlling the expression of matrix metalloproteinases

(MMPs)

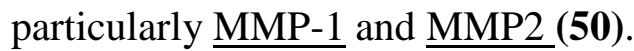

By using ROC curve, RKIP was (Fair) for prediction of distant metastasis of gastric adenocarcinoma. However, in a study it was revealed that AUC of RKIP was 0.93 (Excellent) for prediction of metastasis of clear cell renal cell carcinoma (51).
The current study did not show a statistically significant correlation between CXCR4 and RKIP expression in studied gastric premalignant and adenocarcinoma cases $(\mathbf{P}$ value $\mathbf{= 0 . 1 7 8})$.

No similar published data were found about the relation between CXCR4 and RKIP expression in gastric adenocarcinoma, however in a study (52) it was claimed that RKIP expression was negatively correlated with CXCR4 in tumor tissues and cell lines in breast carcinoma.

A group of researchers was able to demonstrate that RKIP inhibits expression of MMP-1 and CXCR4, thus affecting the ability of metastatic cells to create an osteolytic bone environment in metastatic breast cancer (53).

However, in chronic lymphocytic leukemia (CLL), inhibition of RKIP by locostatin led to a decreased expression of the CXCR4 and reduced the migratory capacity of CLL cells toward SDF-1a as demonstrated in 2018 (54).

Conclusion: These results suggested that both CXCR4 might be involved in gastric carcinogenesis and both CXCR4 and RKIP could be considered as independent prognostic markers in gastric carcinoma. 


\section{References:}

1. Bray F, Ferlay J, Soerjomataram I, Siegel RL, Torre LA, Jemal A. Global cancer statistics 2018: GLOBOCAN estimates of incidence and mortality worldwide for 36 cancers in 185 countries. CA Cancer J Clin. 2018;68(6):394-424.

2. EL-Bolkainy M, Akram M, EL-Bolkainy T, Badawy. Pathology of cancer, 5th edition, gastrointestinal cancers 2016;200:204.

3. Van Cutsem E, Sagaert X, Topal B, Haustermans K, Prenen H. Gastric cancer. Lancet. 2016; 388: 2654-64.

4. Plummer M., de Martel C., Vignat J., Ferlay J., Bray F., \& Franceschi, S. (2016). Global burden of cancers attributable to infections in 2012: a synthetic analysis. The Lancet Global Health, 4(9), e609-e616.

5. Balkwill, Fran and Lisa M. Coussens. "An inflammatory link." Nature 431.7007 (2004): 405-406.

6. Granovsky AE, Rosner MR. Raf kinase inhibitory protein: a signal transduction modulator and metastasis suppressor. Cell Res. 2008;18:452-457.

7. Fukayama M, Rugge M, Washigtom MK: Tumors of the stomach, World Health Organization (WHO) Classification of Tumors, Digestive system Tumors, 5th edition; 2019, Tumors of the stomach: 59-104.

8. Rosai L. Rosai and Ackerman's Surgical Pathology. 11th edition. Gastrointestinal tract.2018:615:635.

9. Okuyama Kishima, Oliveira CE, Banin-Hirata BK, Losi-Guembarovski R, Brajão de, Oliveira K et al.
"Immunohistochemical expression of CXCR4 on breast cancer and its clinical significance." Analytical Cellular Pathology 2015 Jun 16, 891020.

10. Chen Y, Lian G, Ou G, Yang K, Chen J, Li H et al., "Inverse association between Bmi-1 and RKIP affecting clinical outcome of gastric cancer and revealing the potential molecular mechanisms underlying tumor metastasis and chemotherapy resistance." Gastric Cancer 19.2 (2016): 392-402.

11. Nikzaban M, Hakhamaneshi MS, Fakhari S, Sheikhesmaili F, Roshani D, Ahsan B, et al. "The chemokine receptor CXCR4 is associated with the staging of gastric cancer." Advanced biomedical research 13 ,2014: p. 16

12. Xia J, Chen N, Hong Y, Chen X, Tao X, Cheng B et al., Expressions of CXCL12/CXCR4 in oral premalignant and malignant lesions. Mediators of inflammation. 2012 Jan 1, 2012.

13. Long P., Sun F., Ma Y., \& Huang, Y. (2016). Inhibition of CXCR4 and CXCR7 for reduction of cell proliferation and invasion in human endometrial cancer. Tumor Biology Jun 1,37(6), 7473-7480.

14. Huang Y, Zhang J, Cui ZM, Zhao J, Zheng Y. Expression of the CXCL12/CXCR4 and CXCL16/CXCR6 axes in cervical intraepithelial neoplasia and cervical cancer. Chinese journal of cancer. 2013 May;32(5):289.

15. Oh YS, Kim HY, Song IC, Yun HJ, Jo DY, Lee HJ et al. "Hypoxia induces CXCR4 expression and biological activity in gastric cancer cells through activation of hypoxia-inducible factor1a." Oncology reports 28.6 (2012): 2239-2246.

16. Kovelskaya A, Osinsky D, Bubnovskaya L, Ganusevich I, Gumenyuk L, Merentsev S, et al., CXCR4 expression in gastric cancer and bone 
marrow: association with hypoxia-regulated indices, disseminated tumor cells, and patients survival. Journal of Cancer Research. 2015;2015.

17. Shan G, Zhu T\& Hu,Wei P. (2018). Molecular background of the regional lymph node metastasis of gastric cancer. Oncology letters, 15(3), 3409-3414.

18. Cheng Y, Song Y, Qu J, Che X, Song N, Fan Y. (2018). The chemokine receptor CXCR4 and cMET cooperatively promote epithelialmesenchymal transition in gastric cancer cells. Translational oncology, 11(2), 487-497.

19. Huang Y, Ye Y, Long P, Zhao S, Zhang L. Silencing of CXCR4 and CXCR7 expression by RNA interference suppresses human endometrial carcinoma growth in vivo. American Journal of Translational Research. 2017;9(4):1896.

20. Arigami T, Natsugoe S, Uenosono $\mathrm{Y}$, Yanagita S, Arima H, Hirata M, CCR7 and CXCR4 expression predicts lymph node status including micrometastasis in gastric cancer. International journal of oncology. 2009 Jul $1 ; 35(1): 19-24$.

21. Schioppa T, Uranchimeg B, Saccani A, Biswas SK, Doni A, Rapisarda A, et al., Regulation of the chemokine receptor CXCR4 by hypoxia. The Journal of experimental medicine. 2003 Nov 3;198(9):1391-1402.

22. Zhao ZR, Dong XZ, Hu Y, Lu YP, Liu P, Zhang L. LncRNA COL1A1-014 is involved in the progression of gastric cancer via regulating CXCL12-CXCR4 axis. Gastric Cancer. 2020 Mar;23(2):260-272.

23. Teng F, Tian WY, Wang YM, Zhang YF, Guo F, Zhao J et al., Cancer-associated fibroblasts promote the progression of endometrial cancer via the SDF-1/CXCR4 axis. Journal of hematology \& oncology. 2016 Dec 1;9(1):8.

24. Liu B, Ying J, Xu Q, Zhang G, Zhu L. The expression of CXCL12 and CXCR4 in gastric cancer and their correlation to lymph node metastasis. Medical oncology. 2012 Sep 1;29(3):1716-1722.

25. Xiang Z, Zhou ZJ, Xia GK, Zhang XH, Wei ZW, Zhu JT et al., A positive crosstalk between CXCR4 and CXCR2 promotes gastric cancer metastasis. Oncogene. 2017 Sep;36(36):51225133.

26. Sun Y, Mao X, Fan C, Liu C, Guo A, Guan S et al., CXCL12-CXCR4 axis promotes the natural selection of breast cancer cell metastasis. Tumor Biology. 2014 Aug 1;35(8):7765-73.Tumor Biology. 2014 Aug 1;35(8):7765-7773.

27. Mortezaee, Keywan. "CXCL12/CXCR4 axis in the microenvironment of solid tumors: A critical mediator of metastasis." Life Sciences (Mar 7,2020): 117534 .

28. Fujita T, Chiwaki F, Takahashi RU, Aoyagi K, Yanagihara K, Nishimura T et al., Identification and characterization of CXCR4-positive gastric cancer stem cells. PloS one. 2015 Jun 25;10(6):e0130808..

29. Wang W, Zhang Y, Liu W, Zhang X, Xiao $\mathrm{H}$, Zhao $\mathrm{M}$ et al., CXCR4 induces cell autophagy and maintains EBV latent infection in EBVaGC. Theranostics. 2020;10(25):11549.

30. Iwasa S, Yanagawa T, Fan J, Katoh R. Expression of CXCR4 and its ligand SDF-1 in intestinal-type gastric cancer is associated with lymph node and liver metastasis. Anticancer research. 2009 Nov 1;29(11):4751-4758.

31. Gao Y, Li C, Nie M, Lu Y, Lin S, Yuan P et al., CXCR4 as a novel predictive biomarker for 
metastasis and poor prognosis in colorectal cancer. Tumor biology. 2014 May 1;35(5):4171-4175.

32. Jiang C, Ma S, Hu R, Wang X, Li M, Tian F et al., Effect of CXCR4 on apoptosis in osteosarcoma cells via the PI3K/Akt/NF- $\kappa \beta$ signaling pathway. Cellular Physiology and Biochemistry. 2018;46(6):2250-2260.

33. He H, Wang C, Shen Z, Fang Y, Wang X, Chen $\mathrm{W}$ et al., Upregulated expression of CXC chemokine receptor 4 is an independent prognostic predictor for patients with gastric cancer. PloS one. 2013 Aug 6;8(8):e71864.

34. Yang M, Zhang N, Zheng Y, Liu J, Lei T, Xu Y. Genetic variations associated with telomere length confer risk of gastric cardia adenocarcinoma. Gastric Cancer. 2019 Nov 1;22(6):1089-99..

35. Yang SF, Ma R, Pan LL, Cao J, Sheng N. RKIP and peroxiredoxin 2 expression predicts the proliferative potential of gastric cancer stem cells. Oncology letters. 2018 Mar 1;15(3):3173-3177.

36. Martinho O, Simões K, Longatto-Filho A, Jacob CE, Zilberstein B, Bresciani C et al., Absence of RKIP expression is an independent prognostic biomarker for gastric cancer patients. Oncology reports. 2013 Feb 1;29(2):690-6.

37. Kim GE, Kim NI, Lee JS, Park MH, Yoon JH. Reduced RKIP Expression is Associated With Breast Neoplastic Progression and is Correlated With Poor Outcomes and Aberrant Methylation in Breast Carcinoma. Applied Immunohistochemistry \& Molecular Morphology. 2017 Aug 1;25(7):46774.

38. Wei H, Gao HQ, Li HB, Qi SJ, Liu WL, Xu L et al., Correlation among RKIP expression, NF$\kappa \mathrm{B}$ p65 levels, and T-lymphocyte subsets in gastric cardia adenocarcinoma. Genet. Mol. Res. 2015 Jan 1;14:16491-6.

39. Abdi E, Latifi-Navid S, Zahri S, Yazdanbod A, Pourfarzi F. Risk factors predisposing to cardia gastric adenocarcinoma: Insights and new perspectives. Cancer medicine. 2019 Oct;8(13):6114-26.

40.Liu H, Li P, Sun P, Zhang J, Wang B, Jia B et al., RKIP suppresses gastric cancer cell proliferation and invasion and enhances apoptosis regulated by microRNA-224. Tumor Biology. 2014 Oct 1;35(10):10095-103.

41. Faloppa, Martinho O, Neto CS, LongattoFilho A, Baiocchi G, da Cunha IW et al., Loss of RKIP expression during the carcinogenic evolution of endometrial cancer. Journal of clinical pathology. 2012 Feb 1;65(2):122-128.

42. Nie F, Cao J, Tong J, Zhu M, Gao Y, Ran Z. Role of Raf-kinase inhibitor protein in colorectal cancer and its regulation by hydroxycamptothecine. Journal of biomedical science. 2015 Dec 1;22(1):56.

43. Zhang XM, Zhou C, Gu H, Yan L, Zhang GY. Correlation of RKIP, STAT3 and cyclin D1 expression in pathogenesis of gastric cancer. International journal of clinical and experimental pathology. 2014;7(9):5902..

44.Zlobec I, Baker K, Minoo P, Jass JR, Terracciano L, Lugli A .Node-negative colorectal cancer at high risk of distant metastasisidentified by combined analysis of lymph node status, vasculainvasion, and Raf-1 kinase inhibitor protein expression. ClinCancer Res. 2008; 14:1438.

45. Ye C, Shen Z, Wang B, Li Y, Li T, Yang Y et al., A novel long non-coding RNA lnc-GNAT1-1 is low expressed in colorectal cancer and acts as a tumor suppressor through regulating RKIP-NF- $\kappa B-$ 
Snail circuit. Journal of Experimental \& Clinical Cancer Research. 2016 Dec 1;35(1):187.

46. Afonso J, Longatto-Filho A, Martinho $\mathrm{O}$, Lobo F, Amaro T, Reis RM et al., Low RKIP expression associates with poor prognosis in bladder cancer patients. Virchows Archiv. 2013 Apr 1;462(4):445-53.

47.Fujimori Y, Inokuchi M, Takagi Y, Kato K, Kojima K, Sugihara K. Prognostic value of RKIP and p-ERK in gastric cancer. Journal of Experimental \& Clinical Cancer Research. 2012 Dec 1;31(1):30.

48.Li DX, Cai HY, Wang X, Feng YL, Cai SW. Promoter methylation of Raf kinase inhibitory protein: A significant prognostic indicator for patients with gastric adenocarcinoma. Experimental and Therapeutic Medicine. 2014 Sep $1 ; 8(3): 844-50$.

49.Li Y, Tian Z, Tan Y, Lian G, Chen S, Chen S et al., Bmi-1-induced miR-27a and miR-155 promote tumor metastasis and chemoresistance by targeting RKIP in gastric cancer. Molecular cancer. 2020 Dec;19(1):1-4.

50.Zaravinos A, Bonavida B, Chatzaki E, Baritaki S. RKIP: A Key Regulator in Tumor Metastasis Initiation and Resistance to Apoptosis. Therapeutic Targeting and Impact: Cancers. 2018 Aug 24;10.
51. Papale M, Vocino G, Lucarelli G, Rutigliano M, Gigante M, Rocchetti MT et al., Urinary RKIP/p-RKIP is a potential diagnostic and prognostic marker of clear cell renal cell carcinoma. Oncotarget. 2017; 8: 40412-24.

52.Zhu GD, Liu F, OuYang S, Zhou R, Jiang FN, Zhang B et al., BACH1 promotes the progression of human colorectal cancer through BACH1/CXCR4 pathway. Biochemical and biophysical research communications. 2018 May 5;499(2): 120-127.

53. Yun J, Frankenberger CA, Kuo WL, Boelens MC, Eves EM, Cheng $\mathrm{N}$ et al., Signalling pathway for RKIP and Let-7 regulates and predicts metastatic breast cancer. The EMBO journal. 2011 Nov 2;30(21):4500-14.

54. Crassini KR, Zhang E, Balendran S, Freeman JA, Best OG, Forsyth CJ et al., Humoral immune failure defined by immunoglobulin class and immunoglobulin $G$ subclass deficiency is associated with shorter treatment-free and overall survival in Chronic Lymphocytic Leukaemia. British Journal of Haematology. 2018 Apr;181(1):97-101.

To cite this article: Mona A. Abo El-Khair, Mohebat H. Gouda, Adel Z. Elseaidy, Magda H. Bakr, Rasha M. Abdrabh. Prognostic Significance of Immunohistochemical Expression of Chemokine Receptor (CXCR4) and RKIP in Gastric Carcinoma and Premalignant Lesions of the Stomach. BMFJ 2022:39(academic issue):19-34. 